\title{
L'usage des technologies numériques pour le développement de compétences multimodales en littératie au $21^{\mathrm{e}}$ siècle The use of digital technologies for the development of multimodal literacy skills in the 21st century El empleo de tecnologías digitales en el desarrollo de competencias multimodales en alfabetismo en el siglo XXI
}

\section{Martine Pellerin}

Volume 45, numéro 2, automne 2017

La littératie, tout au long de la vie

URI : https://id.erudit.org/iderudit/1043530ar

DOI : https://doi.org/10.7202/1043530ar

\section{Aller au sommaire du numéro}

\section{Éditeur(s)}

Association canadienne d'éducation de langue française

\section{ISSN}

1916-8659 (numérique)

\section{Découvrir la revue}

Citer cet article

Pellerin, M. (2017). L'usage des technologies numériques pour le développement de compétences multimodales en littératie au $21^{\mathrm{e}}$ siècle. Éducation et francophonie, 45(2), 85-106. https://doi.org/10.7202/1043530ar
Résumé de l'article

L'omniprésence du numérique dans toutes les sphères de nos activités quotidiennes suscite de nouveaux enjeux éducatifs qui exigent une réflexion critique sur l'émergence de nouvelles compétences en littératie adaptées aux besoins des citoyens qui travaillent dans cette ère numérique (Romero, Lille et Patiño, 2017). À la lumière de ces nouveaux défis éducationnels, cet article présente une étude sur l'apport de l'usage des technologies numériques dans le développement de compétences multimodales en littératie au $21^{\mathrm{e}}$ siècle. Plus précisément, il traite d'une étude réalisée en salles de classe primaire et secondaire en contexte d'immersion française dans l'une des provinces anglophones du Canada et portant sur l'apport de l'usage des technologies numériques (par exemple, les iPod et les tablettes) dans le développement de compétences multimodales en littératie. L’analyse de données numériques, comme des vidéos et des artefacts multimodaux créés à l'aide d'applis (apps) par les élèves, dévoile l'apport des possibilités (affordances, terme anglais) offertes par les nouveaux outils numériques dans le développement de compétences multimodales en littératie. Les résultats indiquent particulièrement la contribution des possibilités offertes par ces outils dans la création de nouveaux modes d'expression, de représentation de la pensée, d'action et d'engagement qui, à leur tour, favorisent le développement de compétences multimodales nécessaires au $21^{\mathrm{e}}$ siècle. Ces résultats sont discutés à la lumière des théories d'apprentissage documentées par la recherche dans le domaine des neurosciences cognitives et par des études antérieures sur le concept de multimodalités.

Tous droits réservés $@$ Association canadienne d’éducation de langue française, Ce document est protégé par la loi sur le droit d’auteur. L’utilisation des 2017 services d’Érudit (y compris la reproduction) est assujettie à sa politique d'utilisation que vous pouvez consulter en ligne.

https://apropos.erudit.org/fr/usagers/politique-dutilisation/ 


\title{
L'usage des technologies numériques pour le développement de compétences multimodales en littératie au $21^{\mathrm{e}}$ siècle
}

\author{
Martine PELLERIN \\ Université de l'Alberta, Campus Saint-Jean, Alberta, Canada
}

\section{RÉSUMÉ}

L’omniprésence du numérique dans toutes les sphères de nos activités quotidiennes suscite de nouveaux enjeux éducatifs qui exigent une réflexion critique sur l'émergence de nouvelles compétences en littératie adaptées aux besoins des citoyens qui travaillent dans cette ère numérique (Romero, Lille et Patiño, 2017). À la lumière de ces nouveaux défis éducationnels, cet article présente une étude sur l'apport de l'usage des technologies numériques dans le développement de compétences multimodales en littératie au $21^{\mathrm{e}}$ siècle. Plus précisément, il traite d'une étude réalisée en salles de classe primaire et secondaire en contexte d'immersion française dans l'une des provinces anglophones du Canada et portant sur l'apport de l'usage des technologies numériques (par exemple, les iPod et les tablettes) dans le développement de compétences multimodales en littératie. L'analyse de données numériques, comme des vidéos et des artefacts multimodaux créés à l'aide d'applis (apps) par les élèves, dévoile l'apport des possibilités (affordances, terme anglais) offertes par les nouveaux outils numériques dans le développement de compétences multimodales en littératie. Les résultats indiquent particulièrement la contribution des possibilités 
offertes par ces outils dans la création de nouveaux modes d'expression, de représentation de la pensée, d'action et d'engagement qui, à leur tour, favorisent le développement de compétences multimodales nécessaires au $21^{\mathrm{e}}$ siècle. Ces résultats sont discutés à la lumière des théories d'apprentissage documentées par la recherche dans le domaine des neurosciences cognitives et par des études antérieures sur le concept de multimodalités.

\section{ABSTRACT}

\section{The use of digital technologies for the development of multimodal literacy skills in the 21st century}

Martine PELLERIN, University of Alberta, Saint-Jean Campus, Alberta, Canada

The omnipresence of the digital in every sphere of our daily activities raises new educational issues that demand critical reflection on the emergence of new literacy skills adapted to the needs of citizens working in this digital age (Romero, Lille and Patiño, 2017). In light of these new educational challenges, this article presents a study on the contribution of the use of digital technologies in the development of multimodal literacy skills in the 21st century. More specifically, it deals with a study conducted in primary and secondary classrooms in a French immersion context in one of the English-speaking provinces of Canada. The study explores the contribution of digital technologies (for example, iPods and tablets) to the development of multimodal literacy skills. The analysis of digital data, such as videos and multimodal artifacts created by students with the help of apps, reveals the contribution of affordances offered by the new digital tools in the development multimodal literacy skills. In particular, the results indicate the contribution of affordances offered by these tools with regards to the creation of new modes of expression, representations of thought, action and commitment, which, in turn, promote the development of the multimodal skills needed in the 21st century. These results are discussed in light of learning theories documented by research in the field of cognitive neuroscience and by previous studies on the concept of multimodality.

\section{RESUMEN}

\section{El empleo de tecnologías digitales en el desarrollo de competencias multimodales en alfabetismo en el siglo XXI}

Martine PELLERIN, Universidad de Alberta, Campus Saint-Jean, Alberta, Canadá

La omnipresencia de lo digital en todas las esferas de nuestras actividades cotidianas suscita nuevos retos educativos que exigen una reflexión crítica sobre el surgimiento 
de nuevas competencias en alfabetismo adaptadas a las necesidades de los ciudadanos que trabajan en esta era de lo digital (Romero, Lille y Patiño, 2017). A la luz de los nuevos retos educativos, este artículo presenta un estudio sobre la contribución del empleo de tecnologías digitales en el desarrollo de competencias multimodales en alfabetismo en el siglo XXI. Más precisamente, se trata de un estudio realizado en las salas de clase de primaria y secundaria en contexto de inmersión en francés en una de las provincias anglófonos de Canadá y aborda la contribución del empleo de tecnologías numéricas (por ejemplo, los iPod y las tabletas) en el desarrollo de competencias multimodales en alfabetismo. El análisis de los datos digitales, como videos y artefactos multimodales creados gracias a aplicaciones (apps) por los alumnos, muestra la contribución de las posibilidades (affordance, en inglés), que ofrecen los nuevos instrumentos digitales en el desarrollo de competencias multimodales en alfabetismo. Los resultados muestran, específicamente, la contribución de las posibilidades que ofrecen dichos instrumentos en la creación de nuevos modos de expresión, de representación del pensamiento, de acción y de compromiso que, por su parte, favorecen el desarrollo de competencias multimodales necesarias para el siglo XXI. Dichos resultados se discuten a la luz de las teorías del aprendizaje documentadas por la investigación en los campos de las neurociencias cognitivas y por los estudios anteriores sobre el concepto de multimodalidad.

\section{INTRODUCTION}

L'évolution rapide des technologies dans notre société dénote un passage de l'utilisation de l'ordinateur stationnaire à l'adoption d'outils technologiques numériques et mobiles tels que les téléphones intelligents et les tablettes tactiles. L'utilisation de ces outils numériques reconceptualise les pratiques de littératie au quotidien. Ces changements de pratiques de littératie dans la vie quotidienne qui intègrent de multiples médias et modalités doivent aussi être pris en ligne de compte dans les pratiques pédagogiques de littératie en salle de classe (Dagenais et Toohey, 2014). L'utilisation des technologies numériques pour le développement des compétences en littératie nécessaires au $21^{\mathrm{e}}$ siècle surpasse ainsi une perspective traditionnelle des technologies à titre de convoyeurs d'information, de pratiques répétitives d'habiletés de base par les élèves (Jonassan, 2000; Romero, Lille et Patiño, 2017). L'utilisation des nouvelles technologies numériques en salle de classe entraîne une évolution du concept de littératie traditionnelle (Oskoz et Elola, 2016; Toohey et al., 2015) qui va au-delà des documents imprimés, y compris l'accès à des textes multimodaux et leur création (Kajder, 2010). En outre, les possibilités (dont le terme émerge de la littérature anglo-saxonne [affordances]) offertes par le numérique contribuent à l'émergence de nouveaux modes de représentation, d'expression, 
d'action et d'engagement de la part des élèves dans le développement des compétences en littératie au $21^{\mathrm{e}}$ siècle.

L'innovation en contexte éducationnel bénéficie de nouveaux éclairages de la recherche apportés par les neurosciences cognitives (Andler et Guerry, 2008; OCDE, 2002) qui nous permettent de mieux comprendre comment fonctionne le cerveau et comment on apprend. Malgré l'émergence de ces nouvelles connaissances scientifiques, l'implantation de changements pédagogiques visant les besoins des élèves en matière de développement des compétences en littératie à l'ère numérique demeure «innovatrice, stimulante et inexplorée dans les salles de classe» (Oskoz et Eloda, 2016, p. 158).

À la lumière de ces nouveaux défis concernant l'usage des technologies numériques pour le développement de compétences en littératie dites $\mathrm{du} 21^{\mathrm{e}}$ siècle, la présente étude s'intéresse à l'apport de l'usage des technologies numériques (par exemple les iPod, les tablettes tactiles) dans le développement des compétences multimodales en littératie. Plus particulièrement, l'étude examine l'apport des possibilités (affordances) offertes par ces nouveaux outils numériques dans la création d'environnements d'apprentissage multimodaux et multisensoriels au service du développement des compétences multimodales en littératie à l'ère numérique.

\section{CADRE THÉORIQUE}

\section{Littératie médiatique et multimodale}

Le concept de multimodalité émerge de la recherche anglo-saxonne et en particulier des travaux de Jewitt et Kress (2003). Pour ces auteurs, le concept de multimodalité est défini comme étant l'ensemble des moyens mis en œuvre pour "comprendre les différentes manières de représentation de connaissances et de la construction du sens» (traduction libre). Le concept de multimodalité mis en avant par ces auteurs a pris son ancrage dans la linguistique systémique fonctionnelle développée par le linguiste Halliday (1978). Toujours selon Jewitt et Kress, la littératie multimodale se concentre sur la conception du discours en étudiant les contributions de ressources sémiotiques particulières (par exemple le langage, le geste, les images) codéployées à travers diverses modalités (visuelles, auditives et somatiques). Elle se concentre également sur l'interaction des ressources et leur intégration dans la construction du sens des textes multimodaux (tels que des publicités, des affiches, des reportages, des sites Web, des films). Des travaux plus récents de ces mêmes auteurs (Jewitt, 2009; Kress, 2010) mettent davantage l'accent sur une analyse critique des textes médiatiques et multimodaux de la part des apprenants.

Les travaux dans le domaine de la littératie médiatique multimodale en contexte francophone au Québec (voir par exemple Lebrun, Lacelle et Boutin, 2012) s'appuient sur 
les assises théoriques proposées par les travaux de Jewitt et Kress. Lebrun et ses collaborateurs (2012) estiment que l'apprentissage des compétences sémiotiques et multimodales réside dans l'analyse et la production de multitextes (l'analyse des BD et des extraits de films, par exemple). De plus, selon ces auteurs, l'acquisition des compétences médiatiques multimodales doit faire avant tout l'objet d'un enseignement formel et explicite.

La recherche sur les approches multimodales en littératie en contexte anglophone témoigne particulièrement des changements qu'entraînent les nouvelles technologies dans la combinaison de différents modes de présentation, de représentation de l'information et de la construction du sens (Dagenais et Toohey, 2014; Toohey et al., 2015).

\section{Les possibilités (affordances) offertes par les technologies numériques}

Les nouvelles technologies numériques contribuent à l'accès à de nouvelles possibilités (affordances), inimaginables auparavant. Ce concept de possibilités offertes par les nouvelles technologies a été exploité en matière d'accessibilité, d'immédiateté, de personnalisation et d'intelligence qu'ils offrent aux utilisateurs (voir Pouezevara, 2012). Une perspective plus écologique du concept de possibilités des technologies émergentes a été aussi proposée par Hartson (2003). Celle-ci fait appel à quatre types de possibilités (ou affordances) : cognitive, physique, sensorielle et fonctionnelle.

Le concept de possibilités (ou affordances) a été exploité en particulier dans le contexte anglo-saxon et notamment en contexte d'apprentissage d'une langue seconde par l'utilisation des nouvelles technologies mobiles, connu sous le nom de $\Omega$ aux technologies mobiles indique que les nouvelles affordances offertes par cellesci contribuent à créer de nouvelles possibilités d'apprentissage ainsi que de nouveaux environnements d'apprentissage qui favorisent davantage l'engagement et la motivation chez les apprenants de langue seconde (voir par exemple Godwin-Jones, 2011; Pellerin, 2014). L'usage des nouvelles technologies pour l'apprentissage en langue seconde se répand et incite un plus grand nombre de chercheurs à explorer le comportement des apprenants lors de leurs interactions avec ces nouveaux outils, et en particulier la façon dont ces nouveaux outils technologiques façonnent les expériences des jeunes apprenants de langue seconde (Lafford, 2009; Pellerin, 2017a).

Des études récentes sur la création d'histoires multimodales dans le contexte de l'anglais langue seconde ou additionnelle (par exemple Toohey et Dagenais, 2015; Oskoz et Eloda, 2016) montrent une ouverture vers l'utilisation des technologies au service de la diversité linguistique en salle de classe et l'engagement actif des apprenants dans la construction de sens en contexte de littératie. Cependant, la recherche sur l'usage des technologies numériques pour le développement de compétences multimodales en littératie en français langue seconde et en contexte francophone minoritaire est encore très limitée. 


\section{La conception universelle de l'apprentissage}

Les changements pédagogiques bénéficient aussi de la recherche et des applications possibles issues de diverses disciplines (OCDE, 2002). Un plus grand désir de mieux cibler les besoins variés et particuliers de tous les élèves en salle de classe a contribué à l'adoption d'approches inclusives, telles que l'approche universelle de l'apprentissage. Cette approche prend ses sources dans la planification en architecture pour l'accessibilité des personnes ayant un handicap ou des limitations physiques. Ainsi, les responsables de la planification, par exemple les architectes, les ingénieurs, se sont rendu compte que certains aménagements physiques pouvaient servir non seulement à des personnes avec des handicaps physiques, mais aussi à d'autres utilisateurs (ministère de l'Éducation de l'Ontario, 2012). Ainsi, l'approche de la conception universelle de l'apprentissage comporte des démarches pédagogiques qui répondent aux diverses caractéristiques des élèves ainsi qu'aux besoins variés et uniques de ceux-ci. La conception universelle de l'apprentissage s'informe aussi de la recherche dans le domaine de la neuroscience qui contribue à une meilleure compréhension $\mathrm{du}$ fonctionnement du cerveau et de la relation entre le cerveau et l'apprentissage (OCDE, 2002). Les retombées de la recherche sur la façon dont fonctionne le cerveau contribuent à rehausser notre compréhension des processus d'apprentissage et permettent d'offrir des pistes pédagogiques qui ont le potentiel de mieux répondre aux besoins variés de nos élèves. Brièvement, la conception de l'apprentissage universel s'appuie sur trois grands principes:

- fournir de multiples moyens de représentation;

- fournir des moyens d'action et d'expression nombreux et variés;

- fournir de nombreux moyens de participation et d'engagement.

\section{MÉTHODOLOGIE}

La méthodologie de recherche adoptée pour ce projet s'inscrit dans un paradigme de recherche qualitative alignée sur des perspectives épistémologiques critiques (Denzin et Lincoln, 2005). Elle fait appel avant tout à des méthodes de recherche de type ethnographique et participatif axées sur les enfants (Greene et Hogan, 2005; Hatch, 2007) et sur des méthodes numériques (Pellerin, 2017a). Les questions suivantes ont guidé le processus d'enquête au départ:

- Comment les possibilités (affordances) offertes par ces nouvelles technologies numériques peuvent-elles contribuer à l'émergence de différents modes de représentation, d'expression, d'action et de construction de connaissances au service de la littératie?

- Comment ces nouvelles possibilités (affordances) contribuent-elles au développement des compétences multimodales liées à la littératie à l'ère numérique? 
Il est important de noter ici que les questions soulevées au début du processus d'enquête ne doivent pas être perçues comme étant des concepts statiques.

\section{Le contexte de la recherche et les participants}

Le projet de recherche a été réalisé en contexte d'immersion française dans l'une des provinces anglophones du Canada. Il a touché un total de 20 enseignantes et enseignants et leurs élèves (plus de 350) du primaire ( $1^{\text {re }}$ à $4^{\mathrm{e}}$ année) et du secondaire ( $7^{\mathrm{e}}$ et $8^{\mathrm{e}}$ années) dans trois différentes écoles urbaines et rurales de cette province.

\section{Protocole de recherche et collecte des données}

L'observation ethnographique et numérique effectuée dans le cadre de ce projet de recherche faisait partie de l'une des quatre étapes du protocole de recherche-action collaborative selon le modèle mis au point antérieurement par l'auteure (voir Pellerin [2011] pour la description détaillée de chacune des étapes du modèle). L'ensemble des quatre étapes du modèle de recherche-action collaborative constituait en soi un cycle du projet. Trois cycles prenaient place pendant la période scolaire qui s'étendait du mois de novembre au mois de mai, et cela, durant deux années consécutives. Chacun des cycles était d'une durée moyenne de six à huit semaines.

L'observation ethnographique et numérique en salle de classe par la chercheuse a été effectuée à la fin de chacun des trois cycles du modèle de recherche-action collaborative. L'observation menée dans chacune des classes par la chercheuse variait de 60 à 180 minutes, selon la disponibilité et l'horaire du personnel enseignant. Les classes et les niveaux variaient aussi d'une année à l'autre selon la disponibilité de celui-ci dans leurs écoles respectives et le choix par les directions des écoles. L'observation ethnographique et numérique était réalisée au moyen d'une tablette tactile (iPad). Lors des périodes d'observation et de documentation numérique (enregistrements audio et vidéo), la chercheuse amorçait aussi de manière informelle des dialogues réflexifs (Pellerin, 2017a) avec des élèves pris en petits groupes ou individuellement. La participation des élèves était volontaire, et seulement ceux qui avaient obtenu une permission écrite de leurs parents pouvaient participer à ces dialogues.

Selon le postulat épistémologique adopté pour cette recherche, la collecte des données implique aussi la collecte des données numériques par les enseignantes et les enseignants et les élèves à l'aide d'outils numériques tels que les iPod et les iPad. Cette documentation numérique comprend des enregistrements numériques (audio et vidéo) et des artefacts numériques réalisés à l'aide d'applications à voix et annotations comme les applis Explain Everything et Puppet Pals. 


\section{Analyse et interprétation des données numériques}

Un processus de codage combiné avec les approches de recherche qualitative proposées par Miles et Huberman (1994) est utilisé pour analyser les différentes données numériques obtenues à la suite de l'observation ethnographique en classe par la chercheuse et de la collecte d'artefacts numériques tirés des activités réalisées en classe par les élèves à l'aide d'outils numériques. Le processus de codage à la fois inductif et comparatif a permis de déterminer des catégories initiales guidées au départ par le cadre théorique; par la suite, des catégories ont émergé de l'analyse même de celles-ci.

Il est à noter que les artefacts numériques créés par les élèves ont été recueillis par les enseignantes et les enseignants à titre de travaux quotidiens dans le cadre d'évaluations formatives et sommatives en contexte authentique de salle de classe. Seuls les travaux pour lesquels les parents et les élèves avaient donné leur permission ont fait l'objet de certaines analyses.

\section{ANALYSE ET RÉSULTATS}

L'analyse des données obtenues par l'observation ethnographique numérique en salle de classe et par la documentation d'artefacts numériques met en lumière l'apport des possibilités (affordances) offertes par les nouvelles technologies numériques dans le développement de compétences multimodales en littératie. Plus spécifiquement, cette analyse nous permet de mieux saisir la contribution des possibilités offertes par ces outils numériques dans la création de nouveaux modes d'expression, de représentation de la pensée, d'actions et d'engagement qui, à leur tour, favorisent le développement de ces compétences multimodales en littératie.

En raison de l'espace restreint dont nous disposons, un nombre limité d'exemples d'activités d'apprentissage sera présenté dans la section suivante. Afin de mieux comprendre la portée du concept de multimodalité en contexte de littératie à l'ère numérique, nous avons opté pour des représentations multimodales des résultats. Ainsi, des captures d'écran de vidéos appuyées par un hyperlien (selon la disponibilité et le droit de diffusion de celles-ci), des photos d'artefacts numériques produits à l'aide des applis par les élèves ainsi que des transcriptions de verbatim de dialogues entre la chercheuse et des élèves ont été privilégiées. Il est à noter que la transcription des verbatim a respecté l'originalité des discours oraux des élèves, y compris leurs erreurs grammaticales ou syntaxiques.

Les résultats révèlent l'émergence de deux types de possibilités offertes par les différents outils numériques qui contribuent au développement des compétences multimodales en littératie à l'ère numérique. Il s'agit des possibilités fonctionnelles d'enregistrement audio et vidéo ainsi que des possibilités modales et multisensorielles. 
Les résultats montrent aussi l'apport de ces types de possibilités multimodales et multisensorielles dans la création de nouveaux modes d'expression, de représentation du sens, d'actions et d'engagement dans le développement des compétences essentielles en littératie, telles que savoir parler et écouter, lire et écrire.

\section{Les possibilités fonctionnelles d'enregistrement audio et vidéo}

Les données collectées à travers ce projet de recherche révèlent que les élèves du primaire ( $1^{\text {re }}$ à $4^{\mathrm{e}}$ année) et du secondaire ( $7^{\mathrm{e}}$ et $8^{\mathrm{e}}$ années) s'approprient de nouvelles possibilités multimodales et fonctionnelles, telles que la voix et l'image, offertes par les outils numériques comme les iPod et les iPad afin de développer leurs compétences essentielles en littératie. L'utilisation conviviale et rapide de ces nouvelles fonctionnalités d'enregistrement audio et vidéo contribue aussi à l'émergence de nouveaux modes d'expression et de représentation de la pensée et d'engagement de la part des jeunes apprenants.

\section{Au service des compétences de communication orale}

De nombreux exemples de situations d'apprentissage ont été observés et documentés. Ils illustrent l'apport des possibilités fonctionnelles d'enregistrement audio et vidéo dans le développement des compétences de communication orale, y compris les situations d'expression, d'interaction et de présentation orale (compétences essentielles inscrites dans les programmes d'études en français langue maternelle et langue seconde en Alberta). Les données révèlent aussi que ces possibilités fonctionnelles favorisent des situations d'apprentissage qui visent le développement et l'utilisation du vocabulaire ainsi que la structuration des idées, de la pensée créative, de la verbalisation des connaissances et de la construction des savoirs.

Les résultats montrent que, dès les premières années du primaire ( $1^{\mathrm{re}}$ à $4^{\mathrm{e}}$ année), les élèves utilisaient les fonctionnalités multimodales comme l'enregistrement de la voix et de l'image disponible sur les iPod et les tablettes. Ainsi, les élèves s'engageaient de manière active dans la création de vidéos de leur production orale (par exemple la description d'images liées à une thématique étudiée en salle de classe), dans une narration à partir d'une séquence d'images avec un début, un milieu et une fin, dans un jeu de rôle favorisant le développement et l'utilisation du vocabulaire et des structures de phrases simples et complexes. 
Exemple 1: Expression orale et développement du vocabulaire

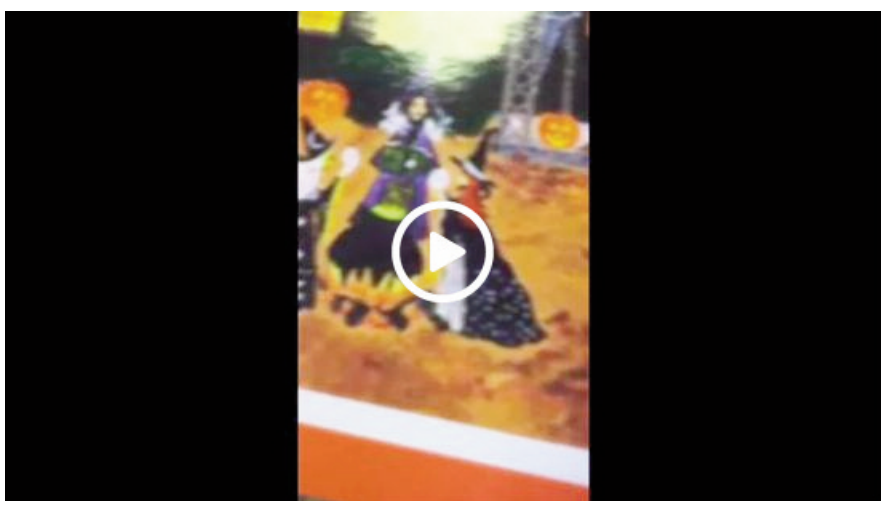

Figure 1. Un élève de $1^{\text {re }}$ année utilise un iPod pour réaliser une vidéo pendant qu'il décrit à voix haute une image représentant une scène $d^{\prime}$ Halloween - thématique étudiée en classe pendant cette période de l'année

(https://capsulesorales.csj.ualberta.ca/index.php/ les-capsules-video/loral-et-les-technologies-numeriques/ pratique-loral-avec-les-ipods/)

Exemple 2: Expression orale, structuration des idées et pensée créative

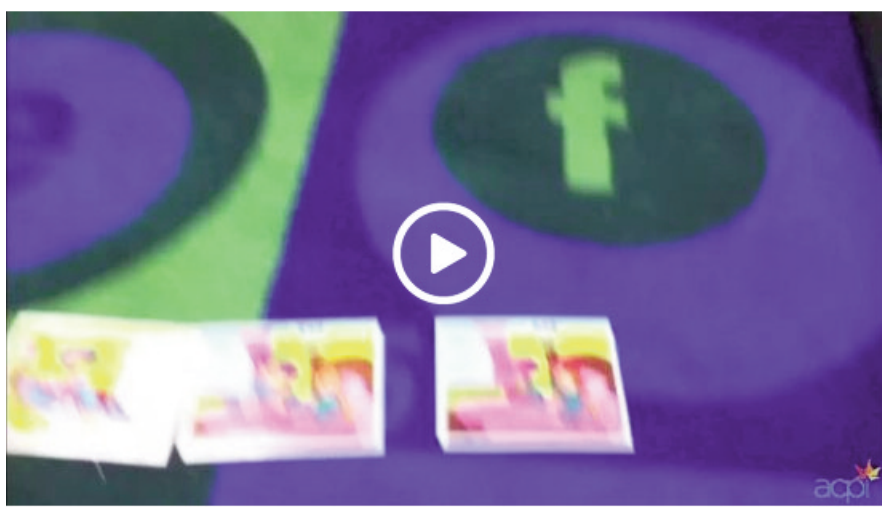

Figure 2. Un élève de $1^{\text {re }}$ année utilise un iPod pour réaliser une vidéo pendant qu'il raconte une histoire à voix haute à partir d'une séquence d'images (https://capsulesorales.csj.ualberta.ca/index.php/ les-capsules-video/loral-et-les-technologies-numeriques/ raconte-avec-le-ipod/)

Exemple 3: Interaction verbale, développement et utilisation du vocabulaire, structuration des idées

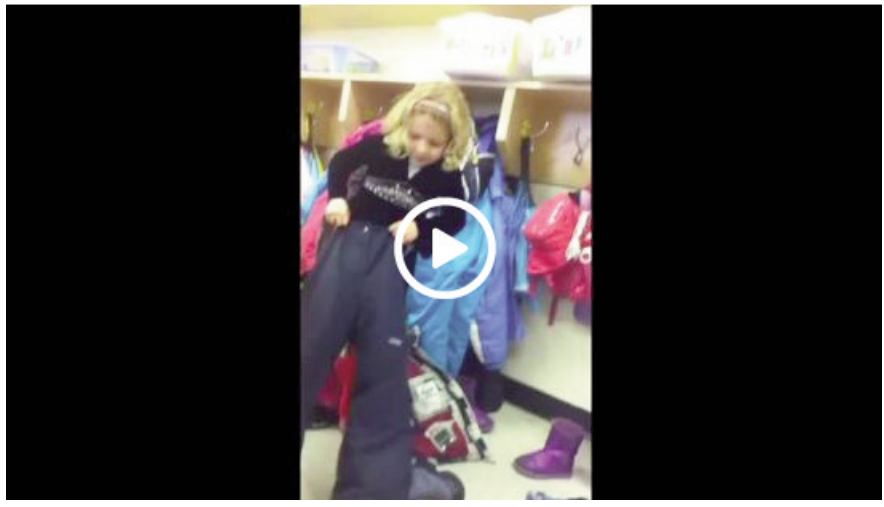

Figure 3. En travaillant avec leurs pairs, des élèves de $2^{\mathrm{e}}$ année créent une vidéo durant un jeu de rôle au sujet des vêtements qu'ils doivent porter I'hiver pour jouer dehors

(https://capsulesorales.csj.ualberta.ca/index.php/les-capsules-video/loral-et-les-technologies-numeriques/raconteavec-le-ipod/) 
Les résultats indiquent également que les élèves du premier cycle du secondaire ( $7^{\mathrm{e}}$ et $8^{\mathrm{e}}$ année) faisaient aussi appel aux fonctionnalités de l'enregistrement de la voix et de l'image disponibles sur les iPod et iPad afin de développer leurs compétences orales interactives. On y retrouve des situations d'apprentissage telles que la création de scénarios de jeux de rôle de type talk-show avec les auteurs et les personnages d'un roman, des débats favorisant le partage de points de vue, de même que des chroniques sur les films, les livres et la musique, pour ne citer que quelques exemples.

\section{Exemple 4: Interaction sociale}

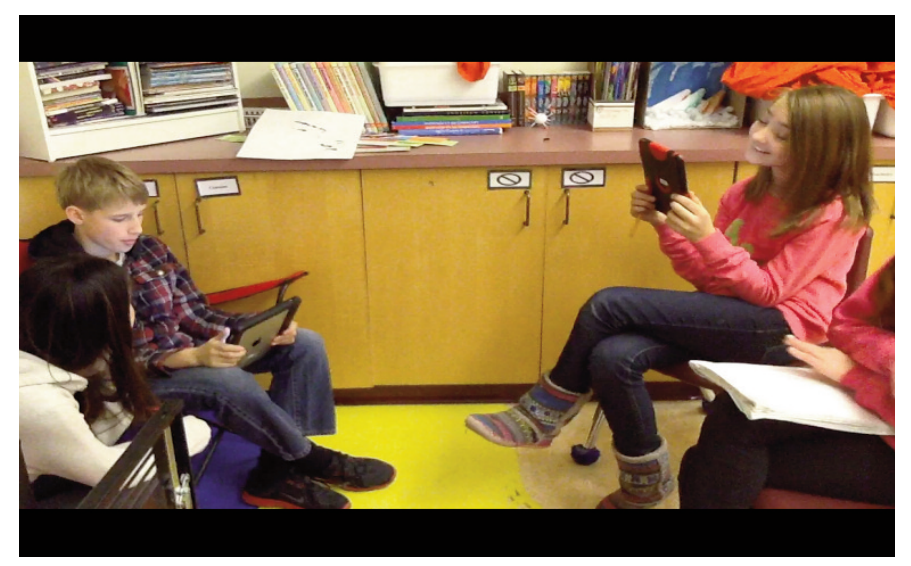

Figure 4. Capture d'écran d'élèves de $7^{e}$ année en train de se filmer à l'aide de l'iPad pendant qu'ils animent une conversation sur leurs choix de musique

\section{Au service des compétences de lecture}

Les résultats montrent que, dès le premier cycle du primaire, les élèves se sont approprié les fonctionnalités d'enregistrement vidéo pour documenter leur lecture à voix haute. L'utilisation conviviale de ces nouvelles fonctionnalités favorise aussi un accès rapide à la visualisation de la vidéo, tout de suite après la création de celle-ci. Dès la première année du primaire, les élèves étaient capables de créer leur propre vidéo et de visualiser celle-ci par la suite, et cela, sans l'aide de leur enseignant ou enseignante.

La possibilité de revenir sur leur lecture à voix haute permet aux élèves de prendre conscience des processus cognitifs déployés lors de l'activité de lecture et de s'engager par la suite dans un processus de réflexion métacognitive sur le déploiement de leurs stratégies de lecture, sur la qualité de lecture en matière de fluidité, de prononciation claire, de ton de la voix, etc. 
Exemple 5: Lecture à voix haute

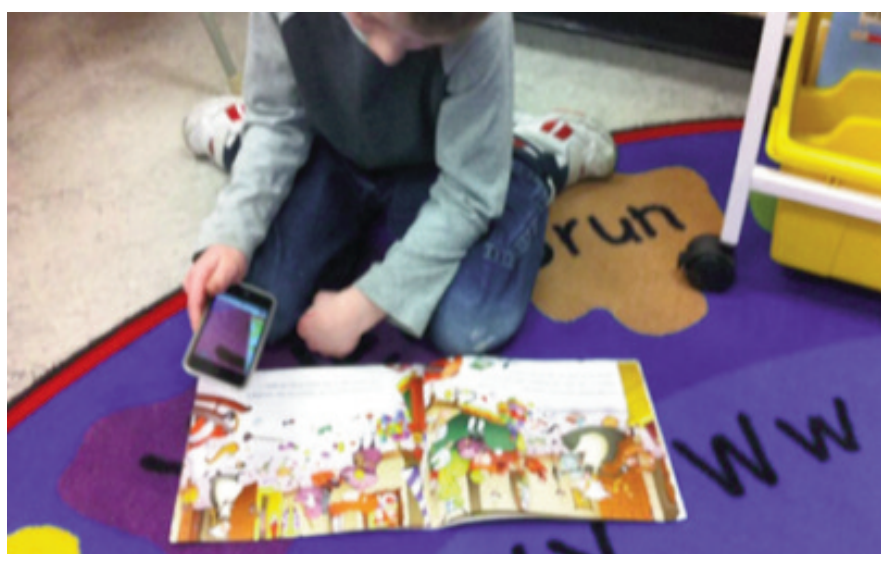

Figure 5. Capture d'écran d'un élève de $1^{\text {re }}$ année qui utilise un iPod pour documenter sa lecture à voix haute

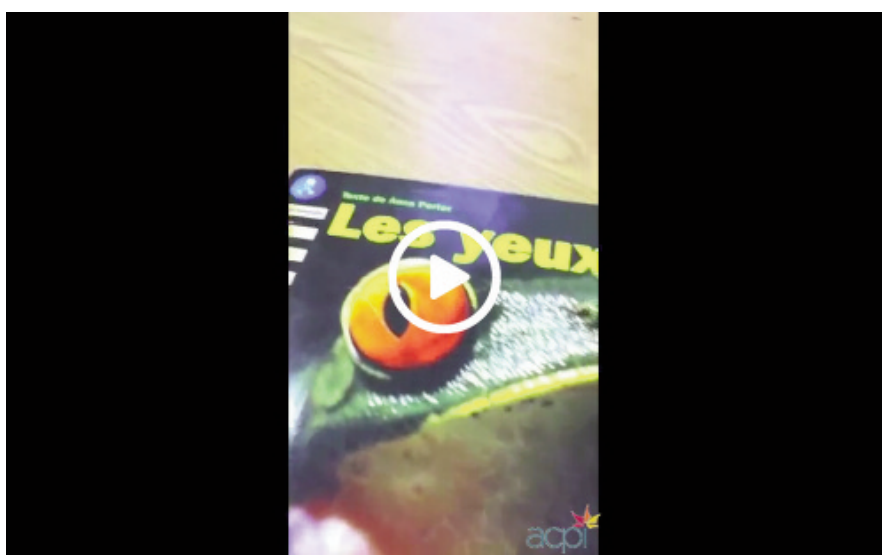

Figure 6. Un élève de $2^{\mathrm{e}}$ année en train de créer une vidéo de sa lecture à voix haute

(https://capsulesorales.csj.ualberta.ca/index.php/ les-capsules-video/loral-et-les-technologies-numeriques/ evaluation-formative-et-sommative-de-la-lecturedocumentation-de-la-lecture-individuelle/)

\section{Au service des compétences à l'écrit}

Les compétences liées à la communication écrite incluent les capacités à communiquer ses idées, ses pensées, ses émotions, ses expériences et ses savoirs ainsi qu'à partager celles-ci avec les autres (ministère de l'Éducation de l'Ontario [MEO], 2006). La communication orale fait également "partie intégrante du processus d'écriture" (MEO, 2006, p. 1.18). Les résultats montrent comment l'oral au service du développement des compétences de l'écrit a été davantage exploré par les élèves grâce aux possibilités fonctionnelles d'enregistrement audio et vidéo offertes par les outils numériques tels que les iPod et les iPad. Grâce à ces nouvelles fonctionnalités, les élèves peuvent enregistrer l'organisation de leurs idées à voix haute afin de mieux planifier leurs idées, ce qui servira à les guider dans la rédaction de leur production écrite. Les nouvelles possibilités fonctionnelles favorisent l'accès rapide à l'enregistrement audio et vidéo qui, à son tour, permet aux élèves de reprendre la rédaction de l'ébauche et de s'engager dans un processus de régulation métacognitive. 


\section{Exemple 6: La communication orale et l'étape de préécriture}

À l'étape de préécriture, un élève de $2^{\mathrm{e}}$ année du primaire dessine un pirate comme personnage principal de l'histoire qu'il planifiait écrire. Il utilise la fonction vidéo sur l'iPod afin d'enregistrer la description à voix haute de son personnage. En regardant sa vidéo, l'élève constate qu'il a oublié un détail dans la description de son personnage. Lorsque la chercheuse lui demande pourquoi il recommence sa vidéo, l'élève explique qu'il a oublié un détail, soit de dire que le chapeau du pirate était noir.

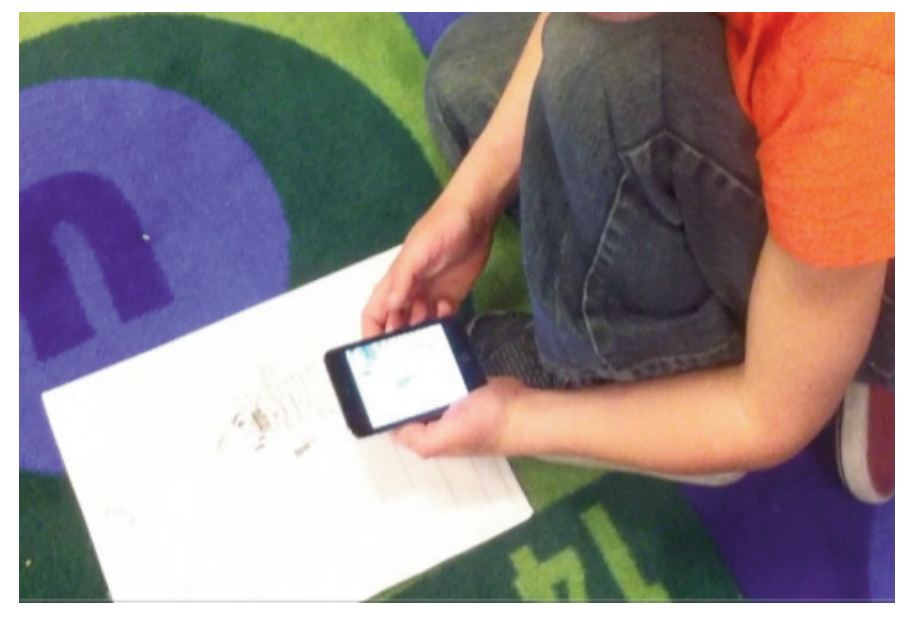

Figure 7. Capture d'écran d'un élève qui enregistre à voix haute la description du personnage principal de son histoire à partir d'un dessin

L'étape de révision d'une première ébauche consiste en une prise de conscience par le scripteur de la qualité et de la clarté du message. La lecture à voix haute joue un rôle important dans cette prise de conscience et plus particulièrement dans l'adoption de stratégies métacognitives d'autorégulation. Les résultats montrent que les possibilités fonctionnelles d'enregistrement audio et vidéo permettent à l'élève d'enregistrer la lecture à voix haute de son ébauche, mais aussi de le visionner afin de s'engager dans un processus de régulation métacognitive envers sa production écrite.

\section{Exemple 7: L'étape de révision en écriture}

À l'étape de la révision, une élève enregistre la lecture à voix haute de son ébauche à l'aide d'un iPod. Par la suite, l'élève écoute l'enregistrement audio de cette lecture de son texte écrit afin de déterminer si elle a besoin d'apporter des changements à celuici ou d'ajouter des détails. 


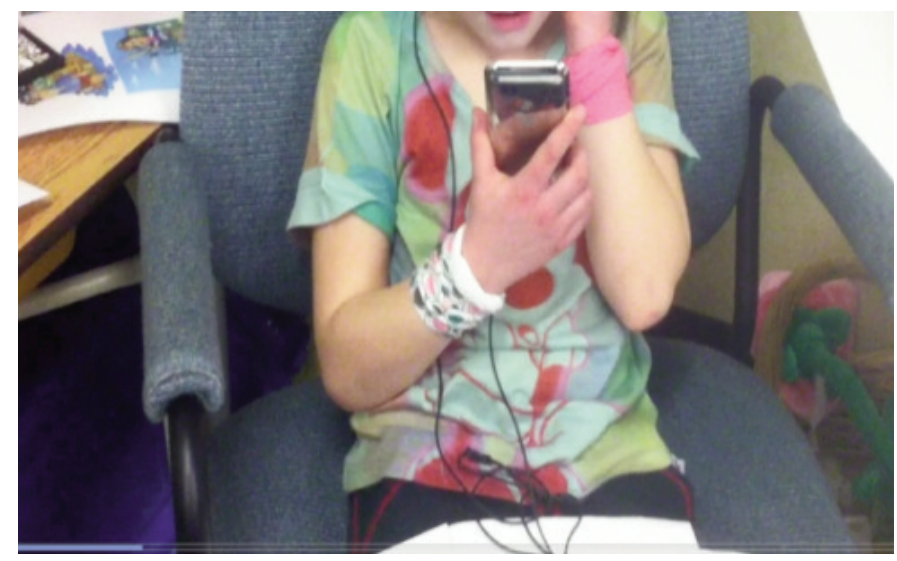

Figure 8. L'élève écoute la lecture à voix haute de son ébauche

\section{Les possibilités multimodales et multisensorielles}

\section{Au service des compétences à l'oral}

Les applications (applis) de création d'animation avec voix, comme Puppet Pals et Toontastics, offrent de nouvelles possibilités sensorielles, inimaginables auparavant. Ces possibilités multimodales et multisensorielles offertes par les applis favorisent la création d'animations qui permettent non seulement l'enregistrement audio de la narration, mais aussi l'ajout d'animations comme celles qu'on trouve dans un spectacle de marionnettes ou dans les dessins animés. Ces applis avec interfaces multimodales incluant l'image et le son offrent aussi de nouvelles possibilités physiques et sensorielles en matière d'actions et d'interactions tactiles avec l'interface même. Les élèves peuvent manipuler les personnages, les faire bouger, changer l'arrière-plan, dessiner, écrire, prendre une photo (de leur ami, par exemple) et l'intégrer par la suite dans l'animation. Toutes ces actions sont aussi enregistrées et elles contribuent à créer un artefact d'animation numérique qui peut être visionné par la suite par l'élève et par l'enseignant et être présenté à la classe et aussi partagé avec les parents à travers le nuage numérique. Les résultats montrent que l'accès à ces multiples modalités et possibilités physiques et sensorielles soutient l'engagement actif des élèves dans des situations de communication orale de manière spontanée et interactive.

Exemple 8: La communication orale spontanée et médiatisée

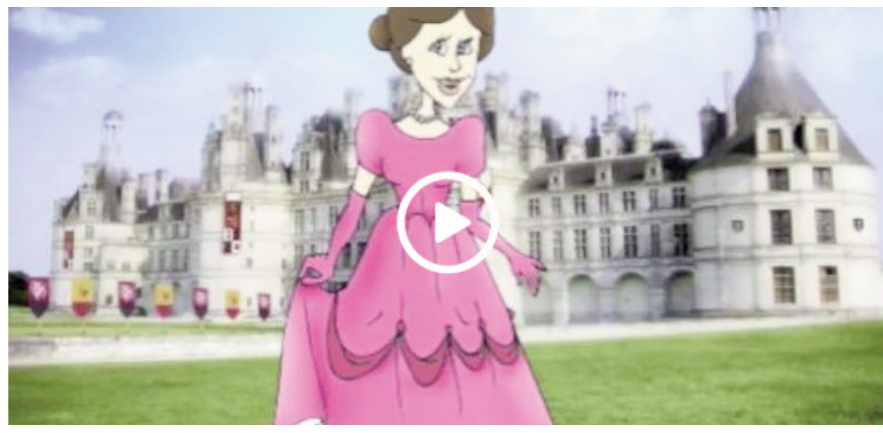

Figure 9. Une animation avec voix réalisée par un élève de $1^{\text {re }}$ année avec l'appli Puppet Pals raconte I'histoire d'une princesse et d'un dragon qui deviennent les meilleurs amis (https://capsulesorales.csj.ualberta.ca/index.php/ les-capsules-video/loral-et-les-technologies-numeriques / raconter-une-histoire-avec-puppetpal-apps/) 
Les résultats tirés de dialogues réflexifs avec des élèves en petits groupes ou individuellement montrent que l'accès à de nouvelles possibilités multimodales et sensorielles offertes par les nouveaux outils numériques leur permet non seulement de s'engager d'une manière active dans les activités de communication orale, mais aussi de développer un sens du contrôle dans la réalisation de la tâche de communication. Les énoncés "tu peux faire...», «tu peux regarder...», que l'on trouve dans l'extrait 1 par exemple, montrent cette capacité d'agir et un savoir-faire de la part de l'élève ainsi que l'émergence d'un sens d'autonomie envers la tâche d'apprentissage même. Voici un extrait d'une conversation entre la chercheuse et un élève de $2^{\mathrm{e}}$ année (7 ans) qui illustre ce sens du contrôle et de l'autonomie de la part de l'élève:

Extrait 1:

- (Chercheuse) Est-ce que tu aimes faire des Puppet Pals?

- (Enfant) Oui.

- (Chercheuse) Pourquoi?

- (Enfant) Parce que tu as les personnes pour jouer avec et tu as les histoires tu peux, tu sais comme les Puppets... Avec les doigts sur le $\mathrm{iPad}$.

- (Chercheuse) Est-ce que cela t'aide à faire de bonnes histoires?

- (Enfant) Oui.

- (Chercheuse) Pourquoi?

- (Enfant) Tu peux faire les bonnes histoires avec les personnes et tu peux regarder l'histoire que tu as faite.

- (Enfant) Tu peux regarder ton histoire que tu as faite avec Puppet Pals... Et tu peux faire encore.

- (Chercheuse) Ah oui... pourquoi?

- (Enfant) Parce que c'est fun.

Les résultats obtenus de l'analyse des artefacts numériques produits avec les applis telles que Puppet Pals montrent aussi que les apprenants s'engagent dans des processus d'autorégulation métacognitive à l'égard de leur production orale multimodale. L'extrait suivant illustre bien comment un élève de $2^{\mathrm{e}}$ année du primaire développe un sens du contrôle lors de la réalisation de son animation et un engagement vis-à-vis de la qualité de sa production multimodale. 
Extrait 2:

- (Chercheuse) Est-ce que tu aimes faire des Puppet Pals?

- (Enfant) Oui.

- (Chercheuse) Pourquoi?

- (Enfant) Parce que tu peux faire les histoires et tu corriges les Puppets et tu peux les tourner.

- (Enfant) Tu peux les tourner aussi...

- (Chercheuse) Est-ce que tu peux faire ça sur un papier?

- (Enfant) Non.

- (Chercheuse) Est-ce que tu écoutes tes Puppet Pals?

- (Enfant) Oui.

- (Chercheuse) Et quand tu écoutes, est-ce que cela t'aide à changer des choses?

- (Enfant) Je regarde mon histoire pour écouter et quand j'ai pas bien fait, je refaire.

- (Chercheuse) Tu vas refaire... cela ne te dérange pas de refaire l'histoire?

- (Enfant) Non.

- (Chercheuse) Pourquoi?

- (Enfant) Parce que c'est juste fun de faire des Puppet Pals et si tu as besoin de refaire, c'est hum, parce que les Puppet Pals sont fun.

- (Chercheuse) Est-ce que tu aimes refaire une histoire sur papier?

- (Enfant) Non.

L'analyse des artefacts numériques produits avec les applis de type tableau interactif, telles que Show Me et Explain Everything, indique aussi que ce type de nouvelles applis offrent des possibilités multimodales et multisensorielles qui n'étaient pas imaginables auparavant. Par exemple, dans la figure 10, l'élève d'une classe de $2^{\mathrm{e}}$ année (7-8 ans) a d'abord pris une photo d'un texte à lire, puis l'a insérée sur le 
tableau interactif de l'appli Explain Everything. Par la suite, l'élève a utilisé la fonctionnalité d'enregistrement de la voix aussi disponible sur l'appli afin de commenter à voix haute la lecture du texte à l'écran. Pendant la lecture, l'élève notait à l'écran tactile (c.-à-d. encerclait ou soulignait) les mots qu'elle éprouvait de la difficulté à décoder ou dont elle comprenait difficilement le sens.

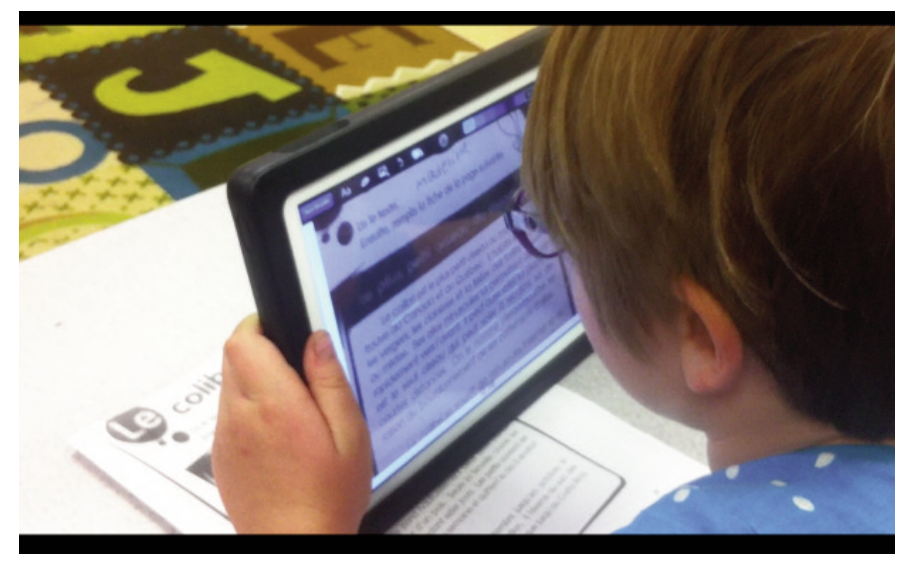

Figure 10. Une capture d'écran d'un élève utilisant I'appli Explain Everything durant une activité de lecture

Les résultats obtenus de dialogues réflexifs avec les élèves indiquent que l'accès aux nouvelles possibilités multimodales et multisensorielles offertes par les nouveaux outils technologiques permet aussi l'adoption par les élèves de stratégies d'autorégulation métacognitive. Ces derniers deviennent plus conscients de leur processus d'apprentissage et font appel de manière autonome à des processus de réflexion métacognitive, tels que l'autorégulation et l'autocorrection, lors de la lecture à voix haute et lorsqu'ils réécoutent celle-ci par la suite.

L'extrait suivant tiré d'une entrevue informelle avec une élève de $2^{\mathrm{e}}$ année du primaire montre le processus de réflexion métacognitive auquel l'élève fait appel pendant l'écoute de l'Explain Everything de sa lecture à voix haute créé à l'aide de l'appli.

Extrait 3:

- J'écoute mon Explain Everything et je dis "hum, je pense que ce mot n'est pas bien", parce que j'écoute les sons comme les syllabes, par exemple ca-li-bri, si je lis calibre, je pense que non parque les syllabes font Co-libre et ça sonne pas bien parce que ça fait de sens parce que calibre, mais colibri fait plus de sens. 


\section{DISCUSSION}

La technologie à elle seule ne transforme pas l'apprentissage; elle contribuerait plutôt à le transformer (Office of Educational Technology, 2017). L'analyse de données numériques en provenance de différentes sources, telles que les enregistrements audio et vidéo et ceux créés à l'aide d'applis (apps) par les élèves, dévoile l'apport des possibilités (affordances) offertes par les nouvelles technologies dans la transformation de l'apprentissage en littératie. De manière plus précise, les résultats montrent que les nouvelles possibilités fonctionnelles, multimodales et multisensorielles contribuent à l'émergence de multiples modes d'expression, de représentation de la pensée et de multiples modes d'action et d'engagement de la part de l'élève qui, à leur tour, favorisent le développement des compétences essentielles en littératie comme le parler, l'écoute, la lecture et l'écrit à l'ère numérique.

Plusieurs constats peuvent être dressés à partir des résultats émergeant de ce projet de recherche. Le premier constat fait par l'auteure est que les possibilités fonctionnelles offertes par les nouvelles technologies permettent aux élèves de s'engager de manière active et autonome dans des activités d'apprentissage qui favorisent le développement des compétences de communication orale incluant les situations d'expression orale et des situations de communication interactive. De plus, les résultats révèlent que ces possibilités fonctionnelles favorisent le développement et l'utilisation du vocabulaire, la structuration des idées, la pensée créative, de même que la verbalisation des connaissances et des processus cognitifs et métacognitifs.

Un deuxième constat est que les nouvelles possibilités fonctionnelles favorisent aussi le développement des compétences en lecture. En particulier, ces nouvelles possibilités multimodales et multisensorielles permettent l'étayage des compétences liées à la fluidité de la lecture, à l'utilisation de stratégies de lecture ainsi qu'à la motivation en matière d'intérêt et d'attitude face à la lecture.

Il convient d'ajouter à ce constat celui du rôle de la communication orale dans le processus d'écriture et la manière dont les élèves s'approprient celle-ci. Grâce aux nouvelles fonctionnalités multimodales, telles que l'enregistrement audio et vidéo, les élèves font appel à de nouveaux modes d'expression et de représentation de la pensée afin de favoriser la planification et l'organisation de leurs idées. Pour certains élèves, l'enregistrement de la verbalisation de leur pensée à l'étape de la préécriture contribue à titre d'étayage au développement de leurs compétences à l'écrit.

Un troisième constat est que l'écoute ou le visionnement des enregistrements audio ou vidéo et des animations créés à l'aide des applis favorise la prise de conscience du processus d'apprentissage, crucial pour le développement des compétences en littératie. À travers les différentes modalités que sont l'audio et la vidéo, les élèves s'engagent dans un processus de régulation métacognitive au regard de leurs productions orales et écrites. Grâce aux nouvelles possibilités qu'offrent les nouvelles technologies, 
l'élève même devient ethnographe de son propre apprentissage en littératie et joue un rôle actif dans l'autorégulation et l'autoévaluation de celui-ci. Ces nouvelles possibilités favorisent aussi le développement d'un sens d'agentivité (contrôle) chez les élèves à l'égard de leur apprentissage; en retour, ces derniers développent un sens de la responsabilité et de l'autonomie au regard du développement de leurs compétences en littératie.

Un quatrième et dernier constat qui se dégage des résultats de ce projet est que les nouvelles possibilités de création de vidéos et d'artefacts numériques d'animation permettent aux élèves de développer des valeurs esthétiques et un sens de l'auditoire. Ainsi, au moment de réaliser les différentes productions d'artefacts numériques, les élèves ont en pensée un auditoire avec lequel ils partageront ces artefacts, ce qui les pousse à se préoccuper de la qualité de leurs productions et de leurs créations. Les élèves deviennent aussi les premiers critiques de leur propre travail et même, parfois, les critiques les plus sévères. Ils sont dès lors d'autant plus conscients de la nécessité d'améliorer leurs compétences en littératie afin d'obtenir un produit final de qualité.

\section{CONCLUSION}

Le concept de littératie multimodale reflète les changements qu'apportent les nouvelles technologies dans la société et, par conséquent, entraîne un changement de paradigme pédagogique concernant le développement des compétences essentielles en littératie à l'ère numérique. Les savoirs - lire, écrire, écouter et parler - demeurent des compétences essentielles dans la société au $21^{\mathrm{e}}$ siècle. Cependant, l'acquisition de ces compétences fondamentales en littératie requiert des innovations pédagogiques qui suscitent le passage d'un modèle de transmission traditionnelle (enseignement magistral) vers une participation active et authentique de l'élève dans son processus d'apprentissage. À son tour, cette participation favorise la prise de contrôle et le développement d'un sens de la responsabilisation et de l'autonomie (Pellerin, 2017b).

L'usage des nouvelles technologies numériques en contexte éducationnel exige avant tout un changement de paradigme de l'apprentissage au $21^{\mathrm{e}}$ siècle, qui va bien au-delà d'un débat réductionniste concernant la valeur ajoutée de l'utilisation des outils technologiques pour l'apprentissage. Chaptal (2008) argumente que la question de l'efficacité des nouvelles technologies «était mal posée» (p. 17), et cela, pour trois raisons:

- elle relève d'une approche étroitement productiviste fondée sur les seules comparaisons mesurables;

- elle se fonde sur l'illusion de la possibilité d'isoler, au sein de la complexité qui caractérise toute situation pédagogique, une variable unique, en l'occurrence les TIC; 
- elle fait l'impasse sur le paradoxe consistant à évaluer l'efficacité supposée de la nouveauté par comparaison avec des indicateurs en cohérence avec les modèles traditionnels (Chaptal, 2008, p. 18).

L’adoption de pratiques pédagogiques qui favorisent le développement des compétences multimodales en littératie est cruciale afin que nos élèves soient des citoyens engagés et critiques à l'ère de la globalisation et du numérique. En guise de conclusion, j'emprunterai les mots de John Dewey, philosophe et réformateur éducationnel (1859-1952), pour réitérer le rôle de l'usage des technologies numériques dans le développement des compétences multimodales pour une littératie adaptée aux besoins du $21^{\mathrm{e}}$ siècle: "If we teach today's students as we taught yesterday's, we rob them of tomorrow» (Si nous enseignons aux étudiants d'aujourd'hui comme nous avons enseigné à ceux d'hier, nous leur volons leur futur - traduction libre).

\section{Références bibliographiques}

ANDLER, D. et GUERRY, B. (dir.). (2008). Apprendre demain. Sciences cognitives et éducation à l'ère numérique. Paris: Hatier.

CHAPTAL, A. (2008). La réalité des TICE: un regard critique. Dans D. Andler et B. Guerry (dir.), Apprendre demain. Sciences cognitives et éducation à l'ère numérique (p. 26-51). Paris: Hatier.

DAGENAIS, D. et TOOHEY, K. (2014). La production vidéo: une pratique multimodale pour tisser des liens entre l'école et les littératies hors scolaires. Nouveaux cahiers de la recherche en éducation, 17(2), 8-31. doi: 10.7202/ 1030886ar

DENZIN, N. K., et LINCOLN, Y. S. (2005). Introduction: The discipline and practice of qualitative research. Dans N. K. Denzin et Y. S. Lincoln (dir.), Handbook of qualitative research (3e éd., p. 1-32). Thousand Oaks, CA: Sage.

DEWEY, J. (1944). Democracy and Education, New York, NY: Macmillan Company

GODWIN-JONES, R. (2011). Mobile apps for language learning. Language Learning \& Technology, 15(2), 2-11. Repéré à http://Itt.msu.edu/issues/june2011/emerging.pdf

GREENE, S. et HOGAN, D. (dir). (2005). Researching children's experience: Approaches and methods. Londres: Sage.

HALLIDAY, M.A.K. (1978). Language as social semiotic. Londres: Edward Arnold. 
HATCH, J. A. (dir.). (2007). Early childhood qualitative research. New York, NY: Routledge.

HARTSON, H. R. (2003). Cognitive, physical, sensory, and functional affordances in interaction design. Behaviour \& IT, 22, 315-338. doi: 10.1080/01449290310001592587

JEWITT, C. (2009). An introduction to multimodality. Dans C. Jewitt (dir.), The Routledge handbook of multimodal analysis (p. 14-27). Londres: Routledge,

JEWITT, C. et KRESS, G. (dir.). (2003). Multimodal literacy. New York, NY: Peter Lang.

JONASSEN, D. H. (2000). Computers as mindtools for schools: Engaging critical thinking. Upper Saddle River, NJ : Prentice Hall.

KAJDER, S. (2010). Adolescents and digital literacies: Learning alongside our students. Urbana, IL: National Council of Teachers of English Publications (NCTE). Repéré à http://www.edu.gov.on.ca/fre/literacynumeracy/paying_attention_ literacy_fr.pdf

KRESS, G. (2010). Multimodality: A social semiotic approach to contemporary communication. New York, NY: Routledge.

LAFFORD, B. (2009). Toward an ecological CALL: Update to Garrett (1991). Modern Language Journal, 93, 673-696.

LEBRUN, M., LACELLE, N. et BOUTIN, J.-F. (dir.). (2012). La littératie médiatique multimodale. De nouvelles approches en lecture-écriture à l'école et hors de l'école. Québec: Presses de l'Université du Québec.

MILES, M. B. et HUBERMAN, A. M. (1994). Qualitative data analysis (2e éd.). Thousand Oaks, CA: Sage.

MINISTÈRE DE L'ÉDUCATION DE L'ONTARIO - MEO. (2006). Guide d'enseignement efficace de l'écriture, de la maternelle à la $3^{e}$ année. Toronto: le Ministère.

MINISTÈRE DE L'ÉDUCATION DE L'ONTARIO - MEO. (2012). Apprentissage par l'action. Littératie intégrée tout au long de la vie. Toronto : le Ministère. Repéré à www.digiexplanations.com/resources/research_1.pdf

OFFICE OF EDUCATIONAL TECHNOLOGY (4 octobre 2017). Technology alone... Repéré à https://twitter.com/officeofEdTech/status/899828577335640064

ORGANISATION DE COOPÉRATION ET DE DÉVELOPPEMENT ÉCONOMIQUES OCDE. (2002). Comprendre le cerveau: vers une nouvelle science de l'apprentissage. Paris: OCDE. 
POUEZEVARA, S. (2012). Mobiles for teaching and learning: Translating theory into practice. Repéré à http://edutechdebate.org/mobile-teaching/mobiles-for-teaching-andlearning-translating-theory-into-practice/

OSKOZ, A. et ELOLA, I. (2016). Digital stories: Overview. Calico Journal, 33(2), 157 173. Repéré à https://journals-equinoxpub-com.login.ezproxy.library.ualberta.ca/index. php/CALICO/article/view/29295/27187

PELLERIN, M. (2011). University-school collaborative action research as an alternative model for professional development through AISI. AISI Journal, 1(1), article 1. Repéré à https://www.uleth.ca/sites/default/files/AISI\%20V1\%201\%201\%20 Fall\%202011.pdf

PELLERIN, M. (2014). Language tasks and mobile technologies: A paradigm shift in designing task-based CALL for young language learners / Activités langagières et technologies mobiles: un changement de paradigme dans la conception des tâches en apprentissage des langues. Canadian Journal of Learning and Technology / La revue canadienne de l'apprentissage et de la technologie, 40(1). Repéré à https://www.cjlt.ca/index.php/cjlt/article/view/26295

PELLERIN, M. (2017a). La pensée médiatisée au service du développement des habiletés de régulation métacognitive chez les élèves. Dans C. Dumais, R. Bergeron, M. Pellerin et C. Lavoie. (dir.), L'oral et son enseignement: pluralité des contextes linguistiques (p. 221-242). Montréal: Peisaj.

PELLERIN, M. (2017b). Rethinking the concept of learner autonomy within the MALL environment. Dans M. Cappellini, T. Lewis et A. Rivens Mompean (dir.), Learner autonomy and Web 2.0 (p. 91-114). Sheffield, R.-U.: Equinox eBooks.

ROMERO, M., LILLE, B. et PATIÑO, A. (dir.). (2017). Usages créatifs du numérique pour l'apprentissage au XXI siècle. Québec: Presses de l'Université du Québec.

STRAUSS, A., et CORBIN, J. (1994). Grounded theory methodology: An overview. Dans N. K. Denzin et Y. S. Lincoln (dir.), Handbook of qualitative research (p. 118). Londres: Sage.

TOOHEY, K. et DAGENAIS, D. (2015). Videomaking as sociomaterial assemblage. Language and Education, 29(4), 302-316. doi: 10.1080/09500782.2015.1006643

TOOHEY, K., DAGENAIS, D., FODOR, A., HOF, L., NUÑEZ-MENDEZ, O., SCHULZE, E. et SINGH, A. (2015). “That sounds so cooool”: Entanglements of children, digital tools and literacy practices. TESOL Quarterly, 49(3), 461-485. doi: 10.1002/ tesq. 236 